\title{
Ellipsometry investigation of nucleation and growth of electron cyclotron resonance plasma deposited silicon films
}

\author{
M. Li, Y. Z. Hu, J. Wall, K. Conrad, and E. A. Irene \\ Department of Chemistry. The University of North Carolina, Chapel Hill, North Carolina 27599-3290
}

(Received 9 November 1992; accepted 1 March 1993)

\begin{abstract}
The nucleation and initial growth of Si films is fundamental to the understanding and control of rapid thermal chemical vapor deposition (RTCVD), and plasma enhanced CVD(PECVD) processes. Herein is reported the nucleation and growth of Si deposited on oxidized silicon wafers by electron cyclotron resonance (ECR) PECVD in the $600-700^{\circ} \mathrm{C}$ temperature range, under low pressures, and using both in situ spectroscopic ellipsometry and single wavelength real-time ellipsometry. An island growth and coalescence model is used to interpret the real-time ellipsometry data. Initial nuclei distance and growth parameters are derived. Atomic force microscopy was used to observe the early stage of nucleation. In situ spectroscopic ellipsometry is used to measure the final Si film thicknesses and optical properties. The deposited Si films were characterized by cross-sectional transmission electron microscopy.
\end{abstract}

\section{INTRODUCTION}

The study of Si film nucleation and growth has been ongoing for decades. ${ }^{1-9}$ Ellipsometry techniques used in various environments such as low pressure and plasma enhanced chemical vapor deposition, $\mathrm{LPCVD}$ and $\mathrm{PECVD}$, respectively, have been extensively utilized to study the Si nucleation and growth behavior, particularly for amorphous silicon (a-Si) for photoelectronics device applications. ${ }^{5-8}$ Hottier and Cadoret $^{9}$ used single wavelength ellipsometry to study polycrystalline $\mathrm{Si}$ (poly-Si) nucleation on $\mathrm{Si}_{3} \mathrm{~N}_{4}$ a decade ago. The recent trend toward selective deposition for integrated circuit (IC) applications with poly-Si's importance in the IC field, has refocused attention on the area of Si film nucleation and growth. Our approach is to use in situ ellipsometry to examine Si film nucleation resulting from rapid thermal ( $\mathrm{RT}$ ) and electron cyclotron resonance (ECR) plasma processes with potenrial application to Si selective epitaxy growth (SEG). ${ }^{10}$

Both RT and ECR plasma CVD have been shown to be useful in SEG applications. ${ }^{11-13}$ RTCVD employs low thermal exposure, while ECR is preferred for large area uniformity and low temperature deposition. In this work, we show Si film nucleation and growth in ECR plasma system. The work on RTCVD will be reported separately.

\section{EXPERIMENT}

Si films were deposited onto $n$-type, $\langle 100\rangle$ Si crystal wafers which were thermally oxidized to have about $24 \mathrm{~nm}$ thick $\mathrm{SiO}_{2}$. The temperature and $\mathrm{SiH}_{4}$ partial pressures were varied and the effect on nucleation and growth were monitored.

The details of the ECR plasma and ellipsometer systems can be found elsewhere. ${ }^{14}$ The samples were first heated to desired temperatures by a lamp heater behind the wafer, and ceposition at $400 \mathrm{~W}$ and $2.45 \mathrm{GHz}$ microwave power, in an Ar plasma under ECR conditions with $+5 \mathrm{~V}$ substrate bias was monitored using in situ ellipsometry. A mixture of $3 \% \mathrm{SiH}_{4}$ in $\mathrm{Ar}$ gas was used.
A rotating analyzer spectroscopic ellipsometer, with an angle of incidence of $70^{\circ}$, was used to monitor the film formation in situ. Spectroscopic ellipsometry (SE) data was used for optical modeling and single wavelength ellipsometry at a selected wavelength for real-time measurements. Our real-time ellipsometry was carried out at a wavelength of $365 \mathrm{~nm}(3.4 \mathrm{eV})$, with a data acquisition time of $\sim 1 \mathrm{~s}$. The $\mathrm{SE}$ scans were made in the photon energy range from 2.5 to $5.0 \mathrm{eV}$.

In modeling Si nucleation, the measured ellipsometric $\Psi$ and $\Delta$ data are compared to the calculated values as obtained from models to be discussed below. The nuclei are assumed to grow in a hexagonal network. Upon coalescence of the nuclei, voids may be left under the film at the interface, or on top of the film to represent surface roughness or both. In this approach, a continuous one-film model is used, with film thickness identical to the nuclei height and the film properties constructed from that of bulk $\mathrm{Si}$ and voids(air) by using the Bruggeman effective medium approximation (BEMA) ${ }^{15}$ as

$$
\begin{aligned}
f_{v} & \left(N_{\mathrm{Si}}^{2}-N^{2}\right) /\left(N_{\mathrm{Si}}^{2}+2 N^{2}\right) \\
& +\left(1-f_{v}\right)\left[\left(1-N^{2}\right) /\left(1+2 N^{2}\right)\right]=0,
\end{aligned}
$$

where $f_{v}$ is the volume fraction of $\mathrm{Si}$ in the film calculated based on the geometric model, $N_{\mathrm{Si}}$ and $N$ are the complex refractive indices for $S i$ and the film, respectively. The optical data for $\mathrm{Si}$ at high temperature was obtained from our SE measurements of $a-S i$ and $c$-Si. In order to prevent crystallization of $a$-Si, lower temperatures $\left(\sim 600^{\circ} \mathrm{C}\right)$ were used.

In optical modeling by SE, $\Psi$ and $\Delta$ were converted to the pseudo-dielectric function $\langle\epsilon\rangle$, for a complex surface given as

$$
\langle\epsilon\rangle=\sin ^{2} \phi\left[1+\left(\frac{1-\rho}{1+\rho}\right)^{2} \tan ^{2} \phi\right],
$$

where $\rho=\tan \Psi e^{i \Delta}, \phi$ is the angle of incidence. A nonlinear curve fitting routine was used to it the experimental data both in SE and real-time ellipsometry. 

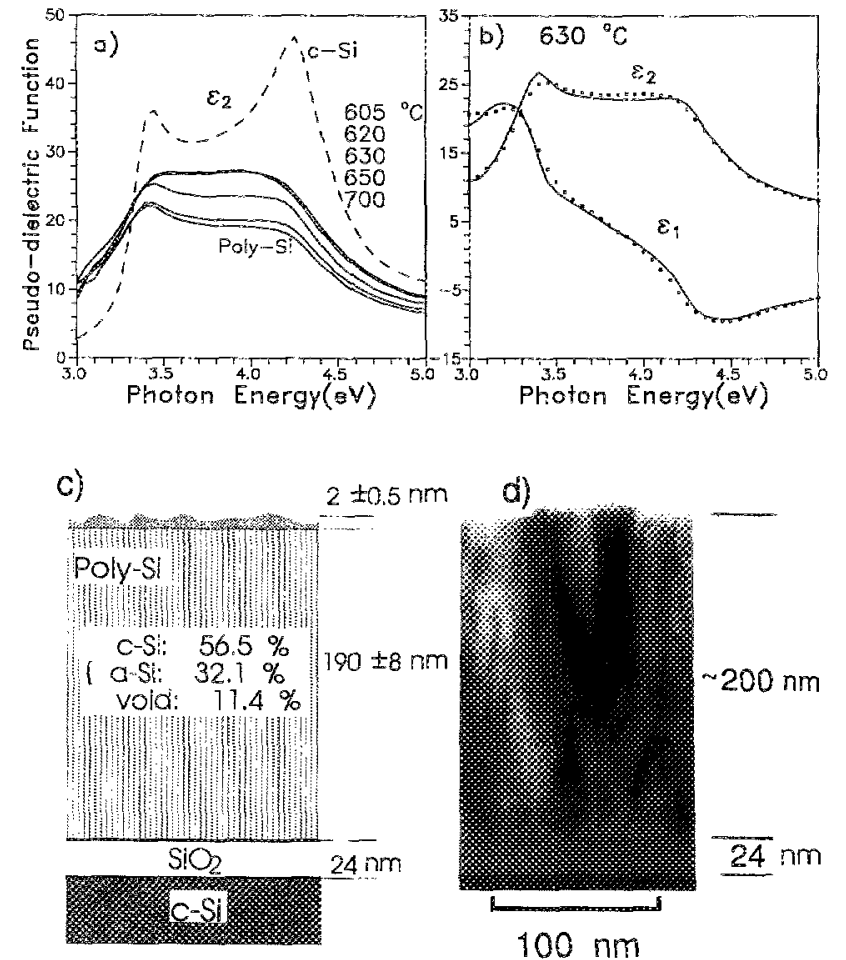

FIG. 1. (a) Pseudo-diejectric function, $\langle\epsilon\rangle$, vs photon energy spectra for ECR PECVD poly-Si films: experimental data for diferent temperatures (solid lines) and a reference spectra for $c$-Si (dashed lines); (b) experimental (dotted lines) and calculated spectra (solid lines) using the optical model shown in (c) for deposited poly-Si film at $630^{\circ} \mathrm{C}$; (c) a schematic optical model; (d) a cross-sectional TEM of the poly-Si film.

\section{RESULTS AND DISCUSSION}

\section{A. The nature of the ECR plasma deposited Si films}

In order to construct a reasonable optical model, the $\mathrm{Si}$ films need to be characterized. The deposited Si films were characterized by $\mathrm{SE}$ and cross-sectional transmission electron microscopy (XTEM). Figure 1(a) illustrates the imaginary part, $\left\langle\epsilon_{2}\right\rangle$, of the pseudo-dielectric function, $\langle\epsilon\rangle$, spectra for ECR PECVD Si films at sample temperatures of 605 to $700^{\circ} \mathrm{C}$. All the spectra show features of $c$-Si which have peaks located at photon energy of 3.4 and 4.2 eV (dashed spectrum). Shown in Fig. 1(b) is the calculated $\langle\epsilon\rangle$ spectra (solid lines) as obtained from the optical model shown in Fig. 1(c), for a Si film grown at $630^{\circ} \mathrm{C}$. The Si film is dominated by $c-\mathrm{Si}$ and $a-\mathrm{Si}$. On top of the $\mathrm{Si}$ film is a roughness layer which is found to be necessary for better data fitting. The roughness layer is considered to be a mixture of $a$-Si, voids, and $\mathrm{SiO}_{2}$. Table I compares ail samples in the roughness layer thickness and Si film compositions for ECR PECVD deposited Si films.

The XTEM result in Fig. 1(d) reveals that the deposited $\mathrm{Si}$ film is poly-Si which has columnar structure and has a thickness comparable to the calculated resuit from $\mathrm{SE}$ as shown in Fig. 1(c).

The optical model is consistent with XTEM, but the $a$-Si fraction obtained from the model must be due to the large amount of grain boundaries. Overall, the ECR deposited films are uniform, with a thin surface roughness layer,
TABLE I. Comparison of ECR PECVD Si films composition as a function of temperature. (The parameters are within about $7 \%$ error.)

\begin{tabular}{lrrrrrr}
\hline \hline Temperature ( ${ }^{\circ} \mathrm{C}$ ) & & 605 & 620 & 630 & 652 & 700 \\
Roughness layer & & 1.5 & 1.7 & 2.2 & 3.0 & 3.3 \\
thickness (nm) & & & & & & \\
Si Film & $c-S i$ & 50.4 & 53.1 & 56.5 & 56.2 & 58.5 \\
composition (\%) & $a-S i$ & 41.7 & 39.9 & 32.1 & 30.6 & 27.3 \\
& void & 7.9 & 7.0 & 11.4 & 13.2 & 14.2 \\
\hline
\end{tabular}

and have the characteristics of $c$-Si. It is also noticed that the volume fraction of voids in the poly-Si film increased significantly from $7 \%$ to $11 \%$ with a deposition temperature rise from 620 to $630^{\circ} \mathrm{C}$.

\section{B. Nucleation models}

Si growth on an amorphous substrate follows an incubation period, during which $\mathrm{Si}$ atom nuclei form and coalesce. These events are influenced by a number of factors, such as temperature, Si precursor concentration, and substrate condition, etc. In general, the formation of a film follows three modes of growth: ${ }^{16}$ (1) layer-by-layer growth, where nucleation is kinetically unimportant; (2) Volmer-Weber nucleation, where individual island nuclei form and coalescence; and (3) Stranski-Krastanov growth, in which a few monolayers of film are first formed, and then island nucleation follows. Our later discussion of the experimental data will only consider the first two models, since we did not observe Stranski-Krastanov growth.

To represent nucleation, we use two extreme geometric models both of which consider islands that coalesce but with different shapes: (1) hemispherical nucleation model, and (2) cylindrical (or disklike) nucleation model. The hemispherical model is adequate for $\mathrm{a}-\mathrm{Si}: \mathrm{H}$ on an amorphous substrate such as $\mathrm{SiO}_{2}$ and on some metals such as Mo, while the cylindrical model holds for $a-\mathrm{Si}: \mathrm{H}$ on $c-\mathrm{Si}$, and $\mu c$-Si on $c$-Si..$^{5-8}$ The use of a hemispherical cap on top of the cylindrical disk may better represent surface roughness. ${ }^{6}$ Figure 2 shows calculated $\Psi, \Delta$ trajectories for Si nucleation on $\mathrm{SiO}_{2}$ for the two models. For both models $D$ is the distance between the nuclei assuming a hexagonal nuclei array, and $D=0$ simulates layer-by-layer growth and is shown as the dashed line in Figs. 2(a) and 2(b). Figure 2(a) for hemispherical nucieation shows that the radius of the nuciei increases continuousiy until the hemispheres come into contact. The positions in the $\psi, \Delta$ plane where the nuclei come into contact are marked by squares. In Fig. 2(b) which is for cylindrical nucleation, the nuclei grow to form columnar structures until contact, and then grow vertically. In this model, the growth ratio $k$, which is defined as the growth rate in the plane parallel to the surface to the out of plane or vertical growth direction, is assumed to be one. ${ }^{6}$ Both simulations assume that surface roughness evolves from the initial nucleation mode and remains unchanged after coalescence. A constant growth rate and film refractive index $(N=5.0-2.8 i)$ are assumed in both models and shown as the solid lines. Other 

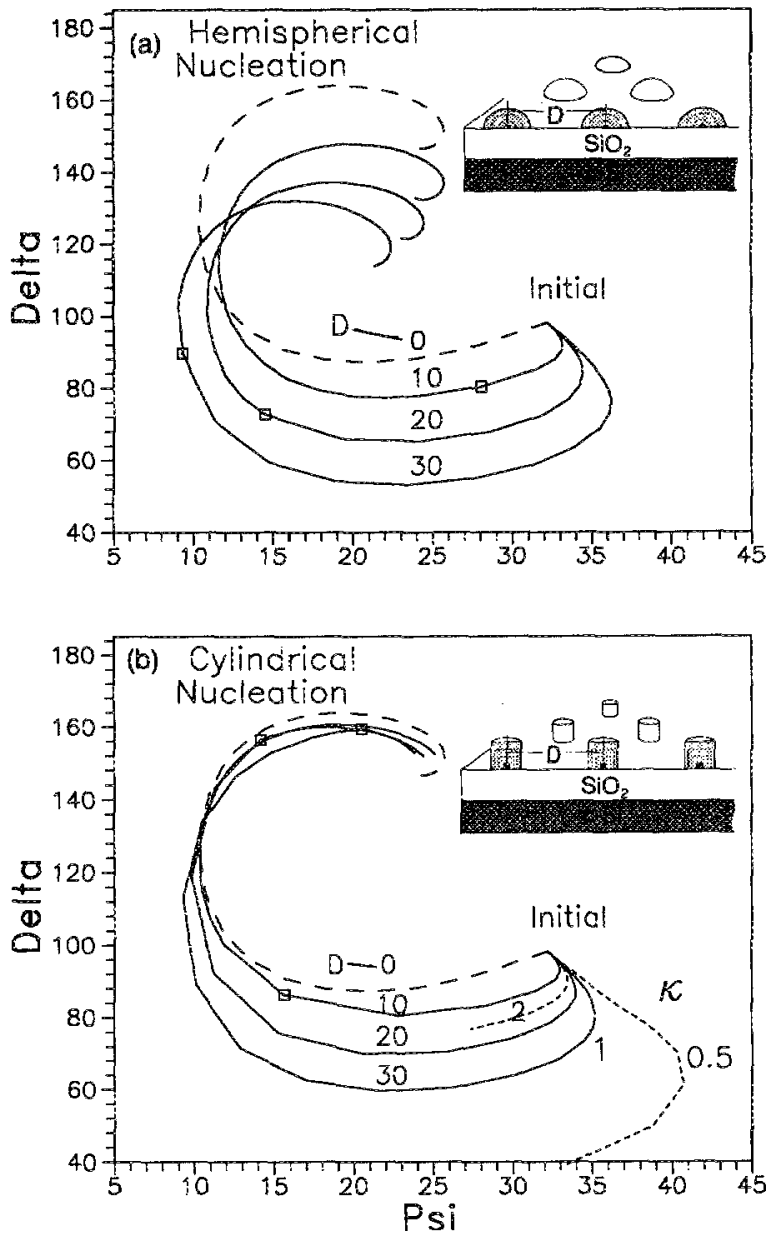

Fir. 2. $\Psi, \Delta$ simulation data assuming a hemispherical nucleation model (a), and cylindrical model (b). The island distance $D$ is varied from 0 to $30 \mathrm{~nm}$. Coalescence of nuclei is marked by squares. The growth ratio $k$ are $0.5,1$, and 2 , respectively.

values of $x$ are also shown in Fig. 2(b) as dashed lines, and $\kappa$ has a significant effect on nucleation. The difference between these two models is mainly in the final film surface morphology, one with hemispherical shape and another disk shape. The treatment of coalescence for cylindrical nucleation is more complicated than for hemispherical model, in that a cone type model was found to be necessary to interpret the data for $\mu c-\mathrm{Si}$ growth on $c-\mathrm{Si}{ }^{6}$

From the simulation, island growth can be clearly distinguished from the layer-by-layer growth from the initial slope of the $\Psi, \Delta$ trajectory. Also, the ellipsometry trajectory provides information about the Si cluster density, as calculated from the initial nuclei distance and geometric distribution of the nucleus at the very early stage of film growth. ${ }^{6}$ The evolution of $\Psi$ and $\Delta$, and the end point provides details about film coalescence and surface roughness.

\section{AFM images of initial nucleation}

As shown above, the nucleation of Si can be evaluated by observing real-time ellipsometric data and our results will be shown and discussed below. However, it is useful to
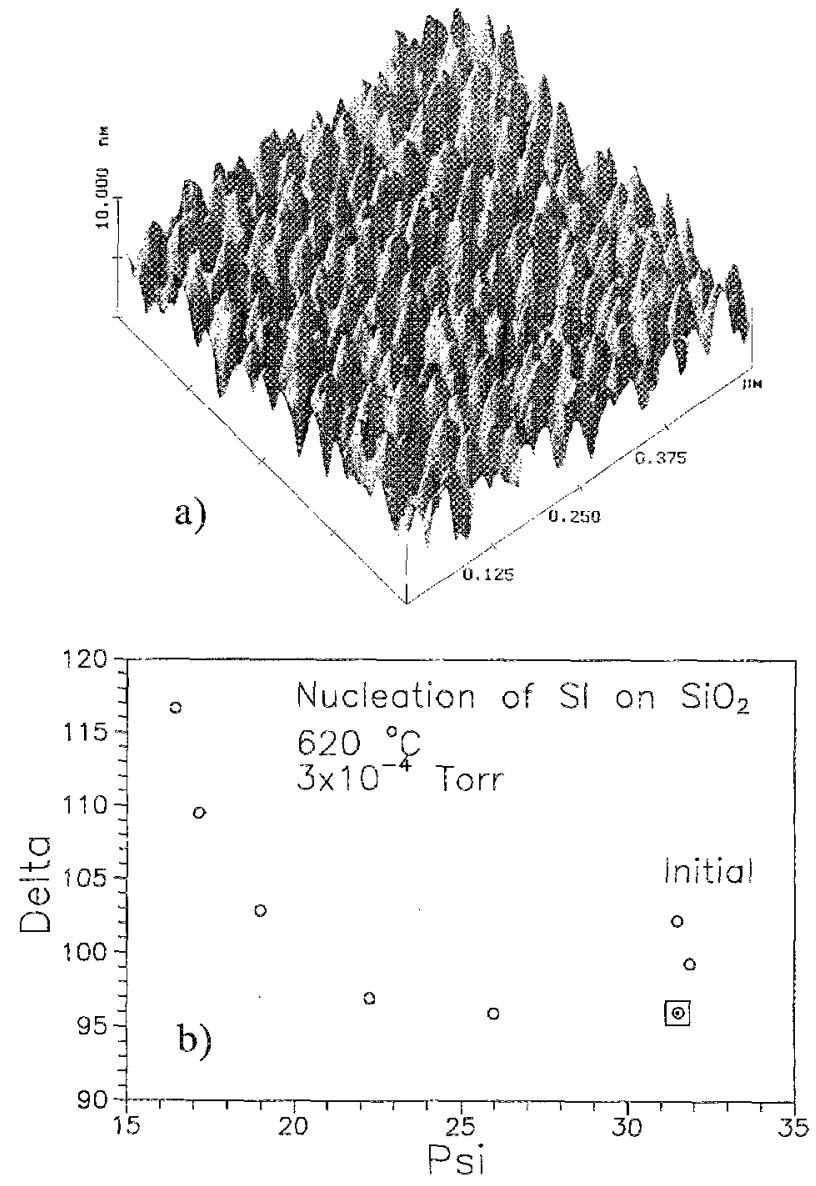

FIG. 3. (a) AFM image of initial nucleation stage of ECR PECVD Si nuclei on $\mathrm{SiO}_{2}$ surface; (b) a corresponding $\Psi, \Delta$ plot showing the position (marked by a square) where AFM image was taken.

use atomic force microscopy (AFM) to image the $\Psi, \Delta$ region which shows nuclei to be present, i.e., prior to coalescence. Figure 3(a) shows an AFM image of the deposited $\mathrm{Si}$ film for a measured $\Delta$ decrease of $\sim 7^{\circ}$, as is shown in Fig. 3(b). Si islands are observed with approximate height of $5 \mathrm{~nm}$ and a separation of approximately $30 \mathrm{~nm}$. We also found that for different temperatures which will be shown to significantly influence Si film growth, the AFM images for the early stages of film nucleation show similar morphologies.

\section{ECR PECVD of poly-Si}

Using the optical models and information from SE, XTEM, and AFM presented above, we follow the real-time evolution of $\mathrm{Si}$ nucleation on $\mathrm{SiO}_{2}$ using single wavelength ellipsometry. The effects of substrate temperature and $\mathrm{SiH}_{4}$ partial pressure on the nucleation and film growth are shown in Figs. 4(a) and 4(b).

\section{Temperature effect}

Figure 4 (a) shows data of real-time $\Psi, \Delta$ trajectories for five different temperatures with $0.06 \mathrm{~min}$ time intervals between data points. The growth at lower temperatures $\left(605\right.$ and $620^{\circ} \mathrm{C}$ ) follow a similar lobe shaped contour, with $\Delta$ decreasing by about $7^{\circ}$, and $\Psi$ increasing by about 

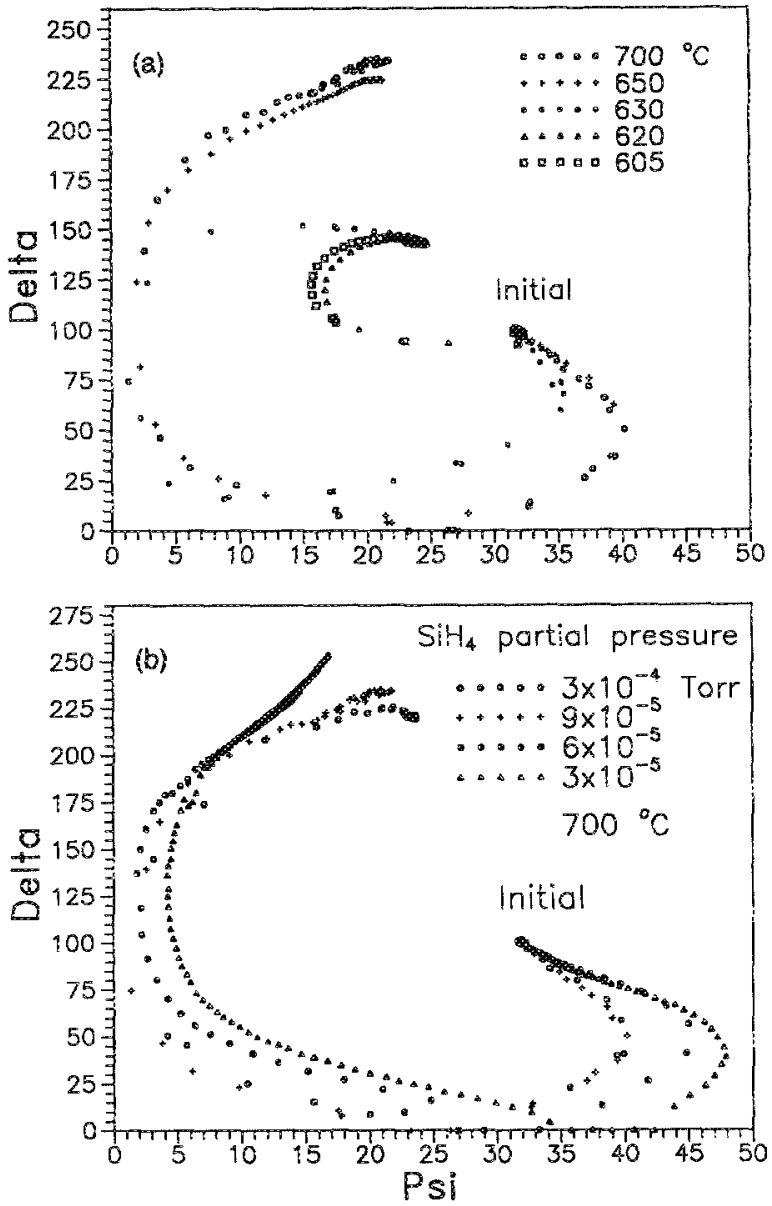

FIG. 4. Real-time single wavelength $\Psi, \Delta$ data for ECR PECVD of poly-Si films: (a) as a function of wafer temperature with $\mathrm{SiH}_{4}$ partial pressure of $9 \times 10^{5}$ Torr and total pressure of $7 \times 10^{-3}$ Torr; (b) as function of $\mathrm{SiH}_{4}$ partial pressure with total pressure in the range of $4 \times 10^{-3}-8 \times 10^{-3}$ Torr at $700^{\circ} \mathrm{C}$.

$0.5^{\circ}$ in the initial region. The $\Psi, \Delta$ trajectory at these temperatures looks like that for $a-\$$ i grown at temperature below $570^{\circ} \mathrm{C}$ (which is not included in the figure). The nucleation and growth at temperatures above $630^{\circ} \mathrm{C}$ is significantly different. At temperatures around $630^{\circ} \mathrm{C}$, a larger lobe shape results from nucleation. After coalescence, $\Psi, \Delta$ return to the opaque condition of $\Psi=24^{\circ}$, $\Delta=145^{\circ}$, which is the same end point for the two lower temperature data sets. The nucleation and growth at 650 and $700^{\circ} \mathrm{C}$ show similar initial nucleation but a different later stage of growth.

Figure 5(a) shows $\Delta$ versus $\Psi$ data for $620^{\circ} \mathrm{C}$. The solid line is a best-fit curve calculated from the cylindrical model using a refractive index $N=4.4-i 3.0$. As mentioned earIier, the $a$-Si and $c$-Si data at high temperatures are measured by SE. However, both $a$-Si and $c$-Si have refractive indices that depend on temperature, and the $a-\mathrm{Si}$ index is dependent on the specific growth procedure. Also, it is never easy to decide whether the $a-\mathrm{Si}$ or the $c-\mathrm{Si}$ index is appropriate for modeling. For this reason, the refractive index chosen in the modeling is from our measurement, although the final value was deduced from curve fitting.
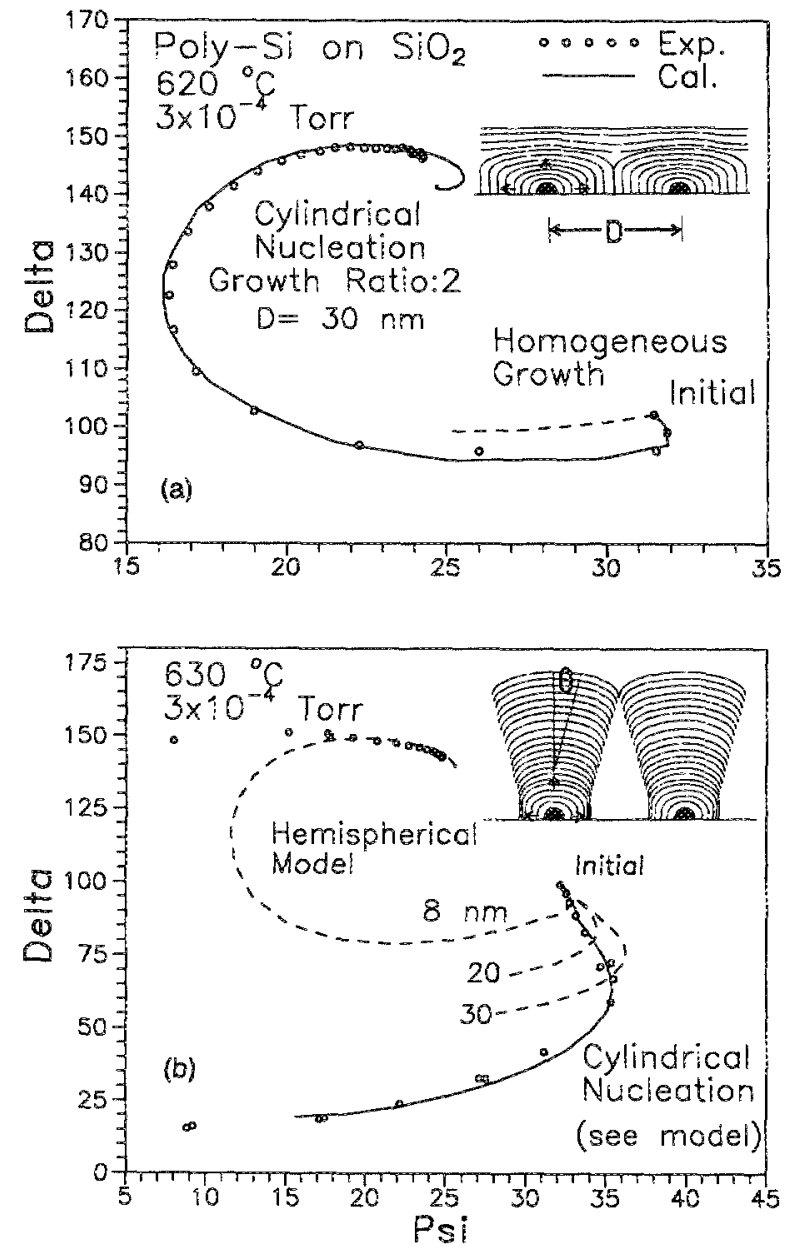

FIG. 5. Nucleation and growth data and fitting for the temperature of $620^{\circ} \mathrm{C}$ (a) and $630^{\circ} \mathrm{C}$ (b) using the cylindrical model with $D=30 \mathrm{~nm}$ (solid line). The dashed line is for homogeneous growth or hemispherical growth.

Also shown for comparison is the homogeneous layerby-layer growth model which is seen to be different from the experimental result. The cylindrical island nucleation model fits the data with an island distance of $30 \mathrm{~nm}$ as determined by AFM [Fig. 3(a)] as a fixed parameter, and with a growth ratio, $\kappa=2$. A schematic of this model is shown in the insert of Fig. 5(a). However, the hemispherical geometry model is also adequate for data fitting, but it yields an island distance $D$ of about $5 \mathrm{~nm}$ which does not agree with the AFM measurement. We expect that in this temperature range, the Si precursors are more likely absorbed on the surface, so that Si nuclei are able to contact to form a continuous film. This is the Kashiev model ${ }^{17}$ by which the deposition is accomplished by the formation of two-dimensional critical nuclei, followed by lateral extension. Upon coalescence of the Si nuclei, a continuous film is formed which progresses in thickness with a thin roughness layer on top.

As seen in Fig. 4(a), the nucleation and growth behavior at $630^{\circ} \mathrm{C}$ is different. From the $\Psi, \Delta$ plot, Fig. $5(\mathrm{~b})$ shows the large change of both $\Psi$ and $\Delta$ in the initial region. This corresponds to nucleation with a large $D$ or sparse nucleation. However, neither the hemispherical nor 
TABLE II. Comparison of the fitting parameters for the cone-like cylindrical model for different temperatures (a) and $\mathrm{SiH}_{4}$ partial pressures (b) for ECR PECVD Poly-Si. (The parameters are within about $10 \%$ error.)

\begin{tabular}{lllll}
\hline \begin{tabular}{c} 
(a) $\begin{array}{c}\text { Temperature } \\
\left({ }^{\circ} \mathrm{C}\right)\end{array}$ \\
\hline$\theta$
\end{tabular} & 620 & 630 & 650 & 700 \\
\hline$S$ & 1.0 & 2.0 & 2.0 & 2.0 \\
$\kappa$ & 2.0 & 1.4 & 1.1 & 1.1 \\
& & & & \\
(b) $\mathrm{SiH}_{4}$ partial & & & & \\
pressure (Torr) & $3 \times 10^{-5}$ & $6 \times 10^{\cdots 5}$ & $9 \times 10^{-5}$ & $3 \times 10^{-4}$ \\
\hline$\theta$ & 0.0 & 2.0 & 2.0 & 2.0 \\
$S$ & 0.57 & 0.57 & 0.6 & 0.6 \\
$\kappa$ & 1.0 & 1.0 & 1.1 & 1.1 \\
\hline \hline
\end{tabular}

the cylindrical-type model fits the data very well over the range of growth. Three dashed lines in the figure are for the hemispherical model with $D=8,20$, and $30 \mathrm{~nm}$, respectively. A conelike model, which is the solid line in Fig. 5 (b) is necessary to fit the initial part of the data. In this mode, the Si nuclei grow with a growth ratio of 1.3. The nuclei grow faster out of plane prior to contact. An angle $\theta$ is introduced to simulate the conical shape of the nuclei and this angle along with the contact factor $S$, the initial nuclei width divided by $D$, determine whether nuclei coalesce to form a continuous film. The physical meaning of $S<1$ from the model ( $S=0.6$ in Table II) is that the nuclei do not coalesce at the interface but rather above, leaving voids. For $S=0.6$, this interface has about $36 \%$ of bulk material and $64 \%$ voids according to this model. Also, we did not observe a cusp feature which indicates surface relaxation. ${ }^{5}$ We note that this feature was observed in our recent RTCVD measurements, but it will not be reported here. We expect that the Si nuclei upon coalescence form a continuous film leaving a surface roughness layer as indicated in Fig. 5(b) using the hemispherical model fitted with $D=8 \mathrm{~nm}$, at the later stage of growth.

We emphasize that our approach for the ECR plasma $\mathrm{Si}$ nucleation and growth model agrees with an earlier morphological study of poly-Si growth at low and high temperatures, ${ }^{j}$ and theoretical calculations of surface morphology in CVD for the slow and fast kinetics. ${ }^{18}$ The deposition temperature is a critical parameter for controlling the initial stage of growth. As our model implies, at lower temperatures the $\mathrm{Si}$ atoms can pile up near the surface and have time to form a layer of nearly uniform concentration. It is different at higher temperatures when the film grows faster in a direction away from the surface. As a result of this and also the surface desorption and diffusion effects, the initial film formation can be regarded as an interface with a lower density film buried under the bulk film.

For higher temperatures of 650 and $700{ }^{\circ} \mathrm{C}$, the initial nucleation can be similarly fitted to the cone model and Table II lists some fitting parameters. However, using our current model we are not able to fit the later stage of growth. From our SE results for these temperatures, we see that the surfaces are rougher and there are larger void fractions in the poly-Si film. We expect that at these tem- peratures, the poly-Si roughness dominates the optical measurement.

\section{2. $\mathrm{SiH}_{4}$ partial pressure}

Figure 4(b) is for the $\Psi, \Delta$ data for different $\mathrm{SiH}_{4}$ partial pressures at $700{ }^{\circ} \mathrm{C}$. The total pressure was in the range of $4-8 \times 10^{-3}$ Torr. We see at this high temperature that clear features of nucleation were observed which varied with $\mathrm{SiH}_{4}$ partial pressure. The initial data were fitted using the same model as in Fig. 5(b) with fitting parameters listed in Table $\mathrm{I}(\mathrm{b})$.

In the model, $\theta=0$ indicates that the film barely coalesces to form a continuous film. When $\mathrm{SiH}_{4}$ partial pressure is low, Si atoms are more likely to form isolated nuclei or crystal facets. Also, it is interesting to observe that increasing the $\mathrm{SiH}_{4}$ partial pressure does not change this behavior very much. From another point of view, whether Si nucleation will form a continuous film or not depends mainly on the surface temperature in our range of experimental conditions.

\section{CONCLUSIONS}

The nucleation and growth behavior of poly-Si in ECR PECVD have been studied using XTEM, AFM, SE, and single wavelength real-time ellipsometry. The data for nucleation and growth of poly-Si from $\mathrm{SiH}_{4}$ on oxidized silicon substrates, can be interpreted using an island nucleation model, where cylindrical-type islands with an initial spacing is in agreement with AFM measurements. The initial spacing, $D$, does not depend strongly on temperature, and is around $30 \mathrm{~nm}$, which is larger than that $(10 \mathrm{~nm})$ for $a$-Si growth as reported by other authors. ${ }^{9}$ Complete coalescence is observed for ECR PECVD at lower temperatures. At higher temperatures, a conelike model has to be used to fit the data.

We have shown how the wafer temperature and gas pressure affect nucleation and growth. Higher temperatures seem to increase the surface roughness as well as increase the growth rate, while the low $\mathrm{SiH}_{4}$ partial pressures can result in discontinuous films.

\section{ACKNOWLEDGMENTS}

The authors thank L. Martin and Dr. D. Maher (North Caroina State University) for XTEM measurements. One of us (M.L.) is grateful for a graduate fellowship from the National Science Foundation (NSF) supported Engineering Research Center at North Caroiina State University. This research is supported by the NSF Engineering Research Center at North Carolina State University.

'J. Bloem, J. Cryst. Growth 50, 581 (1980).

${ }^{2}$ W. A. P. Claasser and J. Bloem, J. Electrochem. Soc. 127, 194 (1980).

${ }^{3}$ M. S. Abrahams, C. J. Buiocchi, R. T. Smith, J. F. Corboy, Jr., J. Blanc, and G. W. Cullen, J. Appl. Phys. 47, 5139 (1976).

${ }^{4}$ N. Matsuo, H. Ogawa, T. Kowzaki, and S. Okada, Appl. Phys. Lett. 60 , 2607 (1992).

${ }^{5}$ R. W. Collins and J. M. Cavese, J. Non-Cryst. Solids 97\&98, 269 (1987).

${ }^{6}$ R. W. Collins and B. Y. Yang, J. Vac. Sci. Technol. B 7, 1155 (1989). 
${ }^{7}$ A. M. Antoine, B. Devillon, and P. P. Cabarrocas, 3. Appi. Phys. 61, 2501 (1987).

${ }^{8}$ A. M. Antoine and B. Drevillon, J. Appl. Phys. 63, 360 (1988).

${ }^{5}$ F. Hottier and R. Cadoret, J. Cryst. Growth 56, 304 (1982).

${ }^{10} \mathrm{~J}$. P. Borland and C. I. Drowley, Solid State Technol. August, 141 (1985).

${ }^{11}$ T. Y. Hsieh, K. H. Jung, D. L. Kwong, T. H. Koschneider, and J. C. Thompson, J. Electrochem. Soc. 139, 1971 (1992).

${ }^{12}$ H. Yamada and Y. Torii, J. Appl. Phys. 64, 702 (1988).
${ }^{13}$ N. Kasai and N. Endo, J. Electrochem. Soc. 139, 1983 (1992).

${ }^{14}$ J. Andrews, Y. Z. Hu, and E. A. Irene, Proc. SPIE 1188, 162 (1989).

${ }^{15}$ D. A. G. Bruggeman, Ann. Phys. (Leipzig) 24, 636 (1935).

${ }^{16} \mathrm{~J}$. A. Venables, G. D. T. Spiller, and M. Hanbuchen, Rep. Prog. Phys. 47, 399 (1984).

17D. Kashchiev, J. Cryst. Growth 40, 29 (1977).

${ }^{18}$ G. S. Bales, A. C. Redield, and A. Zangwill, Phys. Rev. Lett. 62, 776 (1989). 